\title{
EFECTO DE LOS ÁCIDOS NAFTALENACÉTICO E INDOLBUTÍRICO EN EL ENRAIZAMIENTO DE ESTACAS DE Myrciaria dubia (HBK) MC VAUGH, CAMU CAMU
}

\author{
EFFECT OF THE NAFTALENACETIC AND INDLEBUTYRIC ACID IN THE STAKE-ROOTING \\ OF Myrciaria dubia (HBK) MC VAUGH, CAMU CAMU
}

\section{Carlos Oliva Cruz ${ }^{1}$}

\section{RESUMEN}

Entre los meses de setiembre y diciembre de 2003, se instaló el experimento para enraizar estacas de camu camu provenientes de plantas, instaladas en la Estación del IIAP Ucayali hace siete años. Las estacas se instalaron en camas con sustrato de arena y cubiertas con plástico transparente, en cuyo interior a 0,5 m de altura, se protegió horizontalmente con malla de polietileno para amortiguar en un $50 \%$ el efecto directo del sol. El experimento se instaló bajo un diseño de bloque completo aleatorizado con cinco tratamientos y tres repeticiones, donde se utilizó la mezcla de las hormonas enraizantes con ácido indolbutírico (AIB) y ácido naftalenacético (ANA), en dosis de 200 ppm AIB + ANA y 400 ppm AIB + ANA, con dos tiempos de inmersión (24 y 48 horas) para cada dosis y un testigo con cero ppm. Se evaluaron el número y longitud tanto de brotes como de raíces y el porcentaje de enraizamiento a los 60 y 90 días de instalado el experimento. Se encontraron diferencias significativas entre los tratamientos en relación al porcentaje de enraizamiento, y la variable número de raíces estaca se comportó estadísticamente igual. El mejor tratamiento responde a 400 ppm AIB+ ANA en 24 horas de inmersión, seguido por 400 ppm AIB + ANA con 48 horas de inmersión con 55.57 y $40.73 \%$ de enraizamiento respectivamente; por otro lado, el comportamiento del testigo menor con 3.7\% de enraizamiento.

Palabras clave: enraizar, inmersión, dosis, camu camu y estacas, myrciaria dubia.

\begin{abstract}
From September to December of 2003, the stake rooting experiment for camu camu coming from plants was settled, they had been installed for seven years in the IIAP Ucayali station. The stakes settled in sand beds covered with transparent plastic in whose interior at $0.5 \mathrm{~m}$ of height, was protected horizontally with polyethylene mesh to muffle in $50 \%$ the direct effect of the sun. The experiment settled under a design of complete block randomized with five treatments and three repetitions; where the mixture of the hormones enraizantes indlebutyric acid was used (AIB) and the sour naftalenacetic (ELL), in dose of 200 ppm AIB + ANA and 400 ppm AIB + ANA with two times of immersion (24 and 48 hours) for each dose and a witness with $00 \mathrm{ppm}$. It was evaluated the number and so much longitude of buds as of roots and the enraizamiento percentage to 60 and 90 days of having installed the experiment. They were significant differences among the treatments in relation to the enraizamiento percentage, and in the variable roots/stakes number it behaved statistically equally. The best treatment responds to 400 ppm AIB+ ANA in 24 hours of immersion, continued by 400 ppm AIB + ANA with 48 hours of immersion with 55.57 and 40.73 enraizamiento\% respectively, on the other hand the smallest witness's behavior with 3.7 enraizamiento\%.
\end{abstract}

Key words: rooting, immersion, dose, camu camu, myrciaria dubia. 


\section{INTRODUCCIÓN}

El camu camu arbustivo, se encuentra disperso en la cuenca del Amazonas y sus afluentes (Vásquez 2000). Por su alto contenido de vitamina C ( $2800 \mathrm{mg} / 100 \mathrm{~g}$ de pulpa), es considerado como el frutal nativo de gran potencial para la agroindustria y la exportación (Imán 2001). Su propagación es botánica y vegetativa, considerando al método de injertación como el más adecuado según (Riva 1994), agregando que este método nos permite mantener las características genotípicas de la especie y su distribución en los diferentes ecosistemas. Sin embargo se considera que este método de propagación no sería el más recomendable para los trabajos de mejoramiento genético, por considerar el probable efecto del patrón sobre la yema Según Picón \& Acosta (1999) el injerto se utiliza para acortar el tiempo de producción y uniformizar el rendimiento o aprovechar los beneficios de algunos patrones resistentes a enfermedades y plagas.

En cambio en la propagación por estacas, se produce por medio de la réplica del ADN, toda la información genética de la planta madre superior, en efecto, se considera el más adecuado y confiable para los trabajos de mejoramiento genético en la propagación de plantas madres (Vásquez 2000).

El camu camu es una especie en proceso de domesticación, y todavía no se ha definido un método adecuado de propagación por enraizamiento de estacas; diversas investigaciones han reflejado la dificultad de enraizamiento de esta especie y que los mejores resultados se han logrado mediante la aplicación de fitohormonas enraizantes con diferentes dosis.

Santana (1997), realizó enraizamiento utilizando como sustratos arena y aserrín y 0, 200, 2000, 20000 ppm de ANA y toque al 20\% de ANA, bajo cuatro riegos diarios con aspersores. El autor no encontró diferencias significativas entre sustratos, y el porcentaje de enraizamiento fue mayor en 200 y 2000 ppm de ANA con 56 y $48 \%$ respectivamente. Por otro lado, Vásquez (2000), enraizó estacas de camu camu, utilizando tres tipos de diámetro (0,5; 2,0 y mayor de 2,5 cm), sembrados en posición inclinada, con riego diario. Los resultados fueron 0, 10 y 20\% de enraizamiento para los diámetros $0.5,2.0$ y $>2.5 \mathrm{~cm}$ respectivamente, lo que le permitió concluir que a mayor diámetro hay mayor enraizamiento.

A medida que se van avanzando en las investigaciones, los resultados son mejores tal como lo muestra Galucio, P. B. 2002, quienes lograron hasta $90 \%$ de enraizamiento en estacas con diámetros mayores de $0.8 \mathrm{~cm}$, tratados con 200 ppm de ácido naftalenacético (ANA); luego Arévalo (2003), logró hasta 81.48\% de enraizamiento, utilizando estacas de posición basal y diámetro grueso $(2,6$ a $4.0 \mathrm{~cm})$ con riego manual y elevando la temperatura mediante la utilización de plástico transparente. Pero, en las condiciones de Ucayali, todavía no se han logrado buenos resultados, por esta razón es que el presente trabajo de investigación tiene por objetivo evaluar el efecto del ácido naftalenacético y del ácido indolbutírico en diferentes dosis en el enraizamiento de estacas de camu camu, y de alguna manera contribuir a obtener el método adecuado de propagación.

\section{MATERIAL Y MÉTODO}

En los meses de setiembre-diciembre de 2003, en la EE-IIAP Ucayali (Pucallpa) se instaló el ensayo de enraizamiento de estacas de camu camu, mediante la aplicación de hormonas enraizantes, distribuidas en dos tiempos de inmersión. La localización de la estación está en las coordenadas geográficas de 8 22’31" de latitud sur, $74^{\circ} 34^{\prime}$ 35" de longitud oeste, a una altitud de 154 msnm.

El material de propagación fue extraído de plantas establecidas en la EE-IIAP Ucayali, con suelos de textura arcillosa, muy ácidos y con un $\mathrm{pH}$ 4.6; deficiente concentración de fósforo, potasio y boro, toxicidad de aluminio, y normal contenido de materia orgánica, con CIC 13.25 meq/100 g de suelo (López 2002). La zona presenta una temperatura promedio máxima de $34.4^{\circ} \mathrm{C}$, una mínima de $20.2^{\circ} \mathrm{C}$, y una humedad relativa de $82.93 \%$.

La instalación fue bajo un DBCA con cinco tratamientos y tres repeticiones, y se consideró 30 estacas por tratamiento. Los tratamientos se distribuyeron en una mezcla de: 200 ppm (AIB+ANA) con 24 horas, 200 ppm (AIB+ANA) con 48 horas, 400 ppm (AIB+ANA) con 24 horas, 400 ppm (AIB+ANA) con 48 horas y un testigo (cada fitohormona aportaba con 200 ó 400 ppm por tratamiento). Las estacas se cortaron con $25 \mathrm{~cm}$ de longitud 
y 1.5 a 2.0 cm de diámetro, luego se instalaron en camas conteniendo arena como sustrato cubiertas con plástico transparente (un metro de altura), luego dentro de la cama a $0.5 \mathrm{~m}$ de altura colocamos horizontalmente una malla de polietileno para amortiguar en un $50 \%$ el efecto directo del sol. El sistema de riego fue de aspersor nebulizado 3 veces por día (dos minutos de riego).

Las estacas provinieron de los hijuelos de las plantas adultas de camu camu; previa extracción se humedeció un manto de yute, en donde se colocaron las estacas, con la finalidad de evitar la pérdida de humedad durante el transporte hasta la sala de tratamiento con las fotohormonas. En este lugar las fitohormonas de acuerdo a las dosis fueron diluidas en agua destilada, luego las estacas fueron sumergidas en un tercio de su longitud y conservadas según la indicación de los tratamientos.

Las evaluaciones se realizaron a 60 y 90 días posteriores a la instalación del experimento, donde se evaluó: número y longitud de brotes, número y longitud de raíces y porcentaje de enraizamiento.

\section{RESULTADOS Y DISCUSIÓN}

Se obtuvieron los mejores resultados con la aplicación de 400 ppm AIB+ANA en 24 horas y 48 horas de inmersión con enraizamiento de 55.57 y $40.73 \%$ respectivamente. Estos mismos tratamientos muestran superioridad en número y longitud de raíces estacas; con 24 horas de inmersión se obtuvieron 3.11 y $3.36 \mathrm{~cm}$ en raíces y longitud respectivamente y con 48 horas de inmersión se logró 3.29 raíces con 1.39 cm de longitud (figuras 1 y 2). Cuando la dosis fue menor: 200 ppm, el porcentaje de enraizamiento se comportó de modo similar en los dos tiempos de inmersión, pero existió ligera superioridad en relación al número y longitud de raíces por tratamiento, reflejándose en el mayor tiempo de inmersión (48 horas) con 1.30 y 1.11 para número y longitud de raíz respectivamente.

Tabla 1. Comportamiento promedio del porcentaje de enraizamiento, número y longitud de raíces, en enraizamiento de estacas de camu camu, mediante la combinación de dos hormonas enraizantes en la EE-IIAP Ucayali (enero 2004).

\begin{tabular}{lcccccc}
\hline \multirow{2}{*}{ Tratamiento } & \multicolumn{2}{c}{ \% enraizamiento } & \multicolumn{2}{c}{$\mathbf{N}^{\mathbf{0}}$ de raíces } & \multicolumn{2}{c}{ Longitud de raíces } \\
\cline { 2 - 7 } & Promedio & $\begin{array}{c}\text { Desviación } \\
\text { estándar }\end{array}$ & Promedio & $\begin{array}{c}\text { Desviación } \\
\text { estándar }\end{array}$ & $\begin{array}{c}\text { Promedio } \\
(\mathbf{c m})\end{array}$ & $\begin{array}{c}\text { Desviación } \\
\text { estándar }\end{array}$ \\
\hline Testigo & 3.70 & 6.41 & 0.04 & 0.06 & 0.30 & 0.51 \\
$200 \mathrm{ppm}$ AIB + ANA - 24 hr & 18.50 & 6.41 & 0.37 & 0.28 & 0.56 & 0.31 \\
$400 \mathrm{ppm}$ AIB + ANA - 24 hr & 55.57 & 11.15 & 3.11 & 1.90 & 3.36 & 0.93 \\
$200 \mathrm{ppm}$ AIB + ANA - 48 hr & 18.50 & 16.97 & 1.30 & 1.62 & 1.11 & 1.27 \\
$400 \mathrm{ppm}$ AIB + ANA - 48 hr & 40.73 & 12.86 & 3.29 & 2.73 & 1.39 & 0.82 \\
\hline Total & 27.40 & 21.37 & 1.62 & 1.98 & 1.34 & 1.33 \\
\hline
\end{tabular}

En el tratamiento testigo, el porcentaje de enraizamiento es menor en relación a los demás tratamientos con 3.70\% y en número y longitud de raíces no supera la unidad, lo que pone en evidencia que esta especie sin la aplicación de fotohormonas, tiene baja capacidad de enraizamiento; se complementa a esto el bajo número y corta longitud de raíces por estaca, lo que no garantizaría el éxito del prendimiento en campo definitivo.

En relación al número y longitud de brotes por estaca, se observa (tabla 2) que a mayor dosis y tiempos de inmersión se tiene los mejores resultados, resultado que se refleja en 400 ppm AIB + ANA con 48 de inmersión logró 3.67 brotes y 14.93 de longitud, seguido por el tratamiento con dosis 200 ppm AIB + ANA con 48 horas de inmersión que dio 3.26 brotes y 14.16 cm de longitud. Sin embargo, cuando el tiempo de inmersión y la dosis son menores, los resultados tambien son menores, como se observa en 200 ppm AIB + ANA con 24 horas de inmersión con 0.44 brotes y 2.33 cm de longitud. 

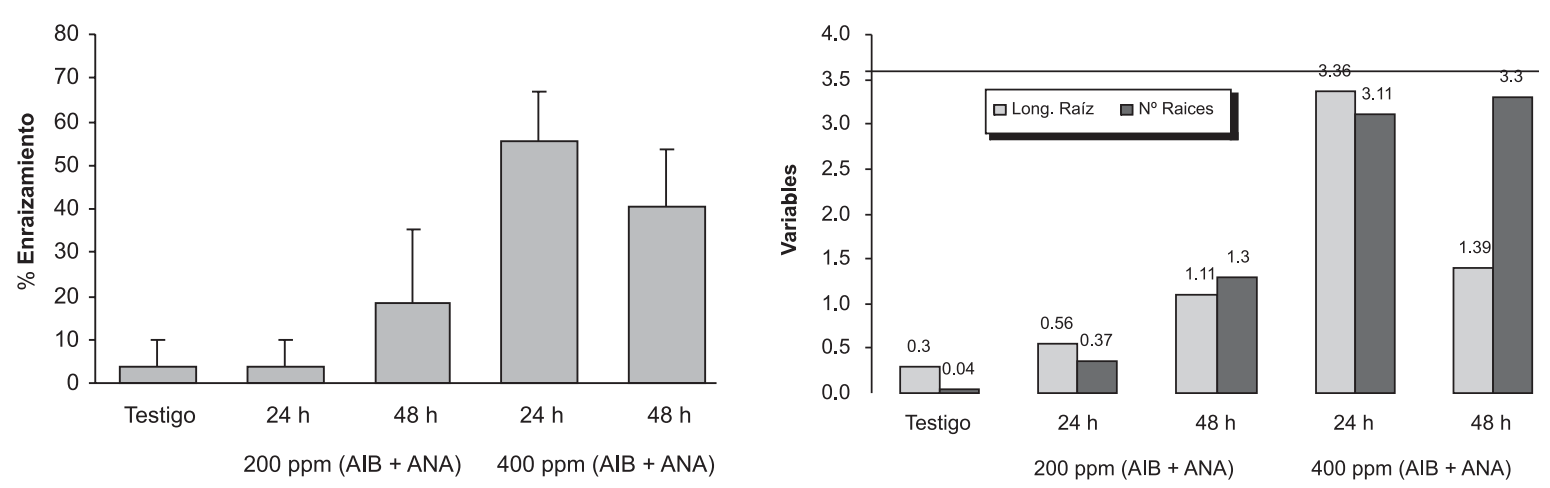

Figuras 1 y 2. Inducción de enraizamiento de estacas de camu camu mediante AIB +ANA.

Tabla 2. Número y longitud de brotes en estacas.

\begin{tabular}{lcccc}
\hline \multirow{2}{*}{ Tratamiento } & \multicolumn{2}{c}{$\mathbf{N}^{\mathbf{0}}$ de brotes } & \multicolumn{2}{c}{ Longitud de brotes } \\
\cline { 2 - 5 } & Promedio & $\begin{array}{c}\text { Desviación } \\
\text { estándar }\end{array}$ & $\begin{array}{c}\text { Promedio } \\
\text { (cm) }\end{array}$ & $\begin{array}{c}\text { Desviación } \\
\text { estándar }\end{array}$ \\
\hline Testigo & 2.15 & 0.93 & 6.97 & 3.63 \\
200 ppm AIB + ANA - 24 hr & 0.44 & 0.45 & 2.33 & 2.22 \\
400 ppm AIB + ANA - 24 hr & 1.44 & 1.02 & 7.20 & 2.87 \\
200 ppm AIB + ANA - 48 hr & 3.26 & 1.52 & 14.16 & 1.88 \\
400 ppm AIB + ANA - 48 hr & 3.67 & 0.40 & 14.93 & 3.70 \\
\hline Total & 2.19 & 1.46 & 9.12 & 5.54 \\
\hline
\end{tabular}

Se encontró diferencias significativas Duncan (0.05) entre tratamientos, distribuidos en tres niveles (tabla 2); se comportó estadísticamente similar la dosis de 400 ppm de AIB+ANA en los dos tiempos de inmersión con 55.5 y $40.7 \%$ de enraizamiento respectivamente. Pero la dosis de 400 ppm de AIB + ANA con 24 horas de inmersión se comportó estadísticamente superior a los demás tratamientos. El tratamiento testigo se comportó estadísticamente similar a los tratamientos con dosis de 200 ppm de AIB + ANA en los dos tiempos de inmersión (tabla 2), resultando ligeramente menor al reportado por Vásquez (2001), quien encontró 10\% de enraizamiento con estacas con $2 \mathrm{~cm}$ de diámetro.

Tabla 3. Prueba estadística de Duncan, en el porcentaje de enraizamiento de estacas de camu camu, bajo la aplicación de dos hormonas enraizantes y dos tiempos de inmersión en la EE-IIAP Ucayali.

\begin{tabular}{lcccc}
\hline \multirow{2}{*}{ Tratamiento } & \multicolumn{3}{c}{ Duncan (Alpha = .05) Subset } \\
\cline { 2 - 5 } & $\mathbf{N}$ & $\mathbf{1}$ & $\mathbf{2}$ & $\mathbf{3}$ \\
\hline Testigo & 3 & 3.700 & & \\
200 ppm AIB+ANA-24hr & 3 & 18.500 & 18.500 & \\
200 ppm AIB+ANA-48hr & 3 & 18.500 & 18.500 & 40.733 \\
400 ppm AIB+ANA-48hr & 3 & & 40.733 & 55.567 \\
400 ppm AIB+ANA-24hr & 3 & & & .153 \\
Sig. & & .169 & 0.053 & \\
\hline
\end{tabular}


Para la variable número de raíces no encontramos diferencias significativas; sin embargo en las variables número de brotes, longitud de brotes y longitud de raíces, sí encontramos diferencias significativas (tabla 4), donde visualizamos que la dosis de 400 ppm AIB+ANA en los dos tiempos de inmersión (24 y 48 horas) reportan los mejores resultados.

Tabla 4. Prueba de medios, para las variables número y longitud de raíces, y número y longitud de brotes en el enraizamiento de estacas de camu camu, mediante la combinación de dos hormonas enraizantes (Duncan, a $=0.05$ ).

\begin{tabular}{lcccc}
\hline \multirow{2}{*}{ Tratamiento } & \multicolumn{3}{c}{ Prueba Estadística de Duncan al 0.05\% } \\
\cline { 2 - 5 } & $\begin{array}{c}\mathbf{N}^{\mathbf{0}} \mathbf{d e} \\
\text { raíces }\end{array}$ & $\begin{array}{c}\text { Longitud raíces } \\
\mathbf{( c m )}\end{array}$ & $\mathbf{N}^{\mathbf{0}}$ de brotes & $\begin{array}{c}\text { Longitud brotes } \\
\mathbf{( c m})\end{array}$ \\
\hline Testigo & $0.04 \mathrm{a}$ & $0.04 \mathrm{~b}$ & $2.15 \mathrm{abc}$ & $6.97 \mathrm{~b}$ \\
$200 \mathrm{ppm}$ AIB+ANA- 24 hr & $0.40 \mathrm{a}$ & $0.37 \mathrm{~b}$ & $0.44 \mathrm{c}$ & $2.33 \mathrm{~b}$ \\
$400 \mathrm{ppm}$ AIB+ANA- 24 hr & $2.98 \mathrm{a}$ & $3.11 \mathrm{a}$ & $1.44 \mathrm{bc}$ & $7.20 \mathrm{~b}$ \\
$200 \mathrm{ppm}$ AIB+ANA-48 hr & $1.30 \mathrm{a}$ & $1.30 \mathrm{~b}$ & $3.26 \mathrm{ab}$ & $14.16 \mathrm{a}$ \\
$400 \mathrm{ppm}$ AIB+ANA-48 hr & $3.29 \mathrm{a}$ & $1.39 \mathrm{~b}$ & $3.67 \mathrm{a}$ & $14.93 \mathrm{a}$ \\
\hline
\end{tabular}

Donde hay letras diferentes en una misma columna, presenta significancia (Duncan 0.05)

En longitud por raíz 400 ppm AIB + ANA con 24 horas de inmersión, se reporta resultados significativamente mejores con $3.11 \mathrm{~cm}$, resultado que no se repite en número y longitud de brotes debido a que los mejores resultados se reflejan en las dosis de 200 y 400 ppm AIB + ANA, ambas con 48 horas de inmersión, siendo mejor el de 400 ppm AIB + ANA, pero sin significancia entre ellas.

Los resultados evidencian la efectividad de las fitohormonas mediante la combinación, con incremento de la temperatura utilizando plástico transparente y malla de polietileno. Al incrementarse la temperatura lo que se ha provocado es acelerar las reacciones bioquímicas, permitiendo actuar de inmediato a las hormonas en la formación de brotes y raíces.

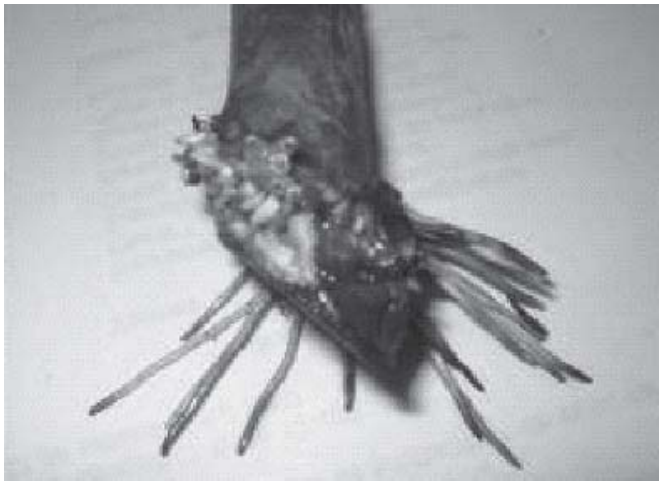

Foto izquierda, enraizamiento con aplicación de hormonas y foto derecha, enraizamiento sin aplicación de hormonas.

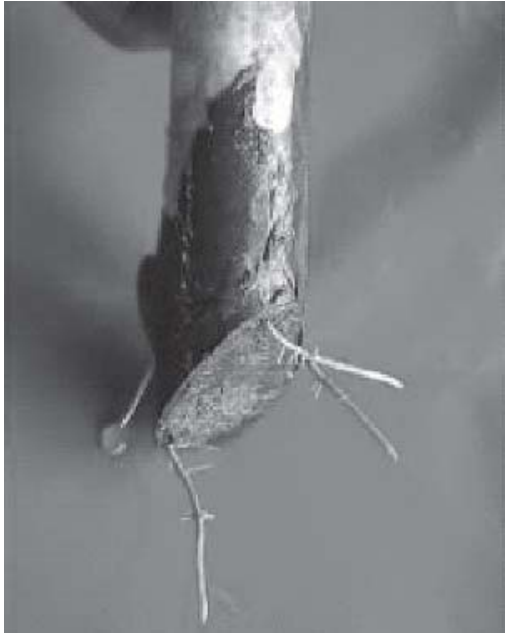




\section{CONCLUSIONES}

- El mejor tratamiento fue con dosis de 400 ppm (AIB + ANA) con 24 horas de inmersión, que se comportó estadísticamente similar a la dosis 400 ppm AIB + ANA con 48 horas de inmersión.

- El tratamiento testigo, obtuvo menor porcentaje de enraizamiento, número y longitud de raíces y número y longitud de brotes.

- El número y longitud de raíces se comportó mejor en 400 ppm AIB + ANA con 24 horas de inmersión.

- No se encontró diferencias significativas entre número de raíces por estaca, pero sí en longitud de raíces por estaca, siendo el mejor tratamiento 400 ppm AIB +ANA con 24 horas de inmersión.

- $\quad$ El mayor número y longitud de brotes se encontró en dosis de 200 ppm (AIB + ANA) y 400 ppm (AIB + ANA), en ambos con 48 horas de inmersión.

\section{BIBLIOGRAFÍA}

ARÉVALO L. 2003. Evaluación de método de clonación de camu camu Myrciaria dubia (H.B.K.) Mc Vaugh, mediante estacas.

BAOS, C.P.; B.F.D. FLOR; C.P. TRUEBA. 1986. Descriptores de camu camu. Programa Nacional de Cultivos Tropicales. (Informe técnico, n. ${ }^{\circ}$ 8). 55 p.

ENCISO, R. 1992. Propagación de Camu-camu (Myrciaria dubia) por injerto. Informe Técnico № 18. Programa de Investigación en Cultivos Tropicales-INIA. Lima. 17 p.

FLORES, P. S. 1997. Cultivo de frutales nativos amazónicos. Manual para extensionistas. TCA. Lima-Perú. 307 p.

GALUCIO, P. B. 2002. Producción de mudas de camu camu (Myrciaria dubia (H.B.K.) Mc Vaugh) por estacas utilizando ramas provenientes de diferentes tipos y posiciones de la planta. Nota Técnica. INPA-Brasil.

HARTMANN y KESTER. 1995. Propagación de plantas. Editorial Continental. México. 760 p.

HUDSON, T. H. y E. K. DALE. 1995. Propagación de plantas. Editorial Continental. México. 733 p.

IMÁN, S. 2001. Cultivo de camu camu Myrciaria dubia H.B.K. en la región Loreto. INIA-SINITTA, Lima-Perú, $32 \mathrm{p}$.

LÓPEZ A.U. 2002. Aplicación de niveles de Calcio, Boro, cobre y Cinz, sobre la productividad del camu camu en suelos aluviales. (Informe trimestral 2003) IIAP Ucayali.

PICON, B.C. y ACOSTA, V.A. (1999) Manual DE los sitemas de producción de camu camu en Selva Baja. Iquitos: Centro de Estudios y Promoción de Tecnologías de Especies Nativas de la Amazonía- Perú.

RIVA, R. 1994. Cultivo del camu camu en Pucallpa. Boletín Técnico. INIA-Pucallpa. 
SANTANA, S. 1998. Propagação de Camu-Camu (Myrciaria dubia (H.B.K.) Mc Vaugh), por meio de estaquia. BIO*Tecnología. www.biotecnologia.com

VÁSQUEZ MATUTE A. (2000) «El Camu camu, Cultivo, Manejo e Investigaciones». Universidad Nacional de la Amazonía Peruana- Facultad de Agronomía- Iquitos-Perú. 